\title{
PROBING THE FUNDAMENTAL EVAPORATION LIMIT WITH A NANOPOROUS MEMBRANE DEVICE \\ Zhengmao Lu ${ }^{l}$, Kyle L. Wilke ${ }^{1}$, Ikuya Kinefuchi ${ }^{2}$, and Evelyn N. Wang ${ }^{1}$ \\ ${ }^{1}$ Massachusetts Institute of Technology, Massachusetts, USA \\ ${ }^{2}$ University of Tokyo, Bunkyo, Japan
}

\begin{abstract}
We designed and fabricated an ultrathin nanoporous membrane device to probe the fundamental limit of evaporation which has remained elusive based on theory and experiments for decades. Our device overcomes the experimental challenge in prior works, including the inability to isolate the interfacial heat transfer resistance and monitor the interface temperature accurately and noninvasively, to characterize kinetically limited evaporation. We showed with both experiments and modeling that kinetically limited evaporation, when normalized properly, is solely determined by the pressure ratio between the ambient and the interface. This has great implications for achieving efficient evaporative transport in practical applications.
\end{abstract}

\section{INTRODUCTION}

Evaporation, a commonly found phenomenon in nature, is extensively used in water desalination [1], steam generation [2], and thermal management [3]. Despite decades of studies [4], the fundamental understanding of evaporation remains limited to date. To recognize the full potential of evaporation, it is desirable to have systems that minimize transport resistances associated with thermal conduction [5, 6], liquid supply [7, 8], and vapor removal $[5,9]$, and maximize interfacial heat transfer, where evaporation can be considered to be kinetically limited. This proved difficult in previous studies [5-14].

Evaporation can be generally driven by the vapor concentration gradient or pressure difference between the interface and the far field. The net molecular flux due to evaporation disturbs the local thermodynamic equilibrium near the interface. This nonequilibrium region, usually a few mean free paths to the interface, is known as the Knudsen layer [15]. Beyond this layer, the disturbance is relaxed by collisions between molecules and the vapor flow regains thermodynamic equilibrium. We define the transport resistance across the Knudsen layer as $R_{\text {kinetic. For air ambient }}$ evaporation, the Maxwell-Stefan diffusion outside the Knudsen layer dominates the overall heat transfer [9] ( $\left.R_{\text {diffusion }} \gg R_{\text {kinetic }}\right)$, which is non-ideal for characterizing $R_{\text {kinetic. To make } R_{\text {kinetic }} \text { the }}$ dominant transport resistance, or to realize kinetically limited evaporation, in this study, we investigated evaporation in pure vapor, where the flow is pressure driven. Based on continuum gas dynamics [16], the pressure loss associated with the gas expansion resistance beyond the Knudsen layer is minimal $\left(P_{\mathrm{K}} \approx P_{\infty}\right)$ for $M_{\mathrm{K}}=$ $u_{\mathrm{K}} / u_{\mathrm{s}} \ll 1$. Here, $P_{\mathrm{K}}$ and $u_{\mathrm{K}}$ are the pressure and bulk velocity of the vapor right outside the Knudsen layer, respectively, $P_{\infty}$ is the far field pressure, and $u_{\mathrm{s}}=\left(\gamma R T_{0}\right)^{1 / 2}$, where $\gamma$ is the adiabatic index, $R$ is the specific gas constant, $T_{0}$ is the interface temperature. This means $R_{\text {expansion }} \ll R_{\text {kinetic. }}$

\section{DEVICE DESIGN}

The interfacial transport can still be possibly limited by the thermofluidic resistance in the liquid. To address this issue, we designed and fabricated an ultrathin nanoporous membrane device (Figure 1a-d). Figure 1a shows the nanodevice design that allows the liquid to wick into the nanopores in the membrane with capillarity, where it is resistively heated by a gold layer and evaporates. The free-standing membrane, connecting two gold contact pads (Figure 1b) is of thickness $t_{\mathrm{m}} \approx 200 \mathrm{~nm}$, made of silicon nitride.

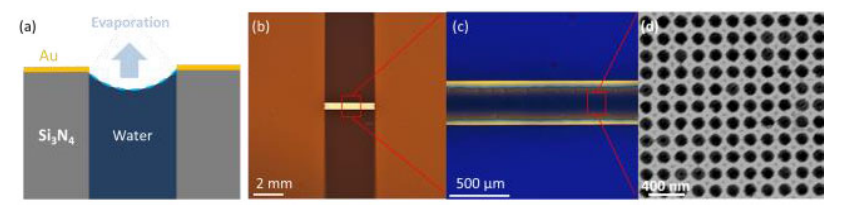

Figure 1: Nanoporous evaporation device. (a) Schematic of evaporation from a nanopore (not to scale). The gold layer is resistively heated to induce evaporation from a pinned meniscus in each nanopore. (b) Image of device with two gold contact pads connected by a free-standing membrane ( $\approx 200 \mathrm{~nm}$ thick). (c) Magnified view of free-standing membrane where the central part is porous and coated with gold. (d) SEM image of the nanoporous membrane with $\approx 140 \mathrm{~nm}$ diameter pores.

The active part of the membrane, located in the middle, is coated with $\approx 40 \mathrm{~nm}$ gold (Figure $1 \mathrm{c}$ ). We defined a nanoporous pattern with pore diameter $d_{\mathrm{p}} \approx 140 \mathrm{~nm}$ and porosity $\approx 0.40$ $(>20,000,000$ pores $)$ in this active area using interference lithography [17] (Figure 1d), while the rest of the membrane is kept non-metallic and impermeable. The combination of small $d_{\mathrm{p}}$ and $t_{\mathrm{m}}$ minimizes the thermal resistance $\left(\sim d_{\mathrm{p}}\right)$ and viscous loss $\left(\sim t_{\mathrm{m}}\right)$ in the liquid and the large number of pores ensures high signal (evaporation) to noise (heat loss) ratios during the experiment. Note that the gold layer also serves as a resistive temperature detector (RTD) which monitors the interface temperature in close vicinity in a non-invasive manner. The other common issue associated with nanoporous configurations is clogging due to non-evaporative contaminants. In the current design, the ultralow thickness of the membrane automatically mitigates the clogging risk. It creates high concentration gradients along the pore with even a small amount of contaminants at the liquid-vapor interface, which drives the contaminants back to the flushing liquid flow underneath the membrane with diffusion. This may have more implications for membrane-based water purification applications. Overall, with evaporation from this ultrathin nanoporous membrane in a pure vapor ambient, we were able to probe the kinetic limit of steady evaporation, which has otherwise been impossible in previous works.

\section{EXPERIMENTS}

To perform characterizations, the device was placed in an environmental chamber to enable controlled vapor temperature and pressure (Figure 2a-b). A custom test fixture interfaced the device to the liquid ports, electrical connections, and facilitates visualization to ensure that liquid did not flood the membrane surface (Figure 2c). 
Prior to the experiment, the liquid reservoir tank was filled with deionized water (Sigma-Aldrich, Water for HPLC) and then heated to $>100{ }^{\circ} \mathrm{C}$ for thermal degassing. The liquid reservoir was subsequently sealed from the ambient. Meanwhile, we calibrated the RTD to an industrial temperature sensor (Omega P-L-A-1/4-6-1/4T-6) in a convection oven (see Supplementary S3). During experiment, the environmental chamber was first pumped down to $<0.5 \mathrm{~Pa}$ (confirmed by MKS 925 Micro PiraniTM vacuum transducer) and then backfilled with pure water vapor from the reservoir. The vapor pressure in the far field was regulated by the chamber wall temperature and measured by a capacitance pressure transducer (MKS 740C Baratron ${ }^{\circledR}$ Manometer). With a peristaltic pump (Masterflex UX-77921-77), the inlet flow rate was maintained at $1 \mathrm{~mL} / \mathrm{min}$. We applied a four-point method to measure the total Joule heating power and read the interface temperature from the RTD.

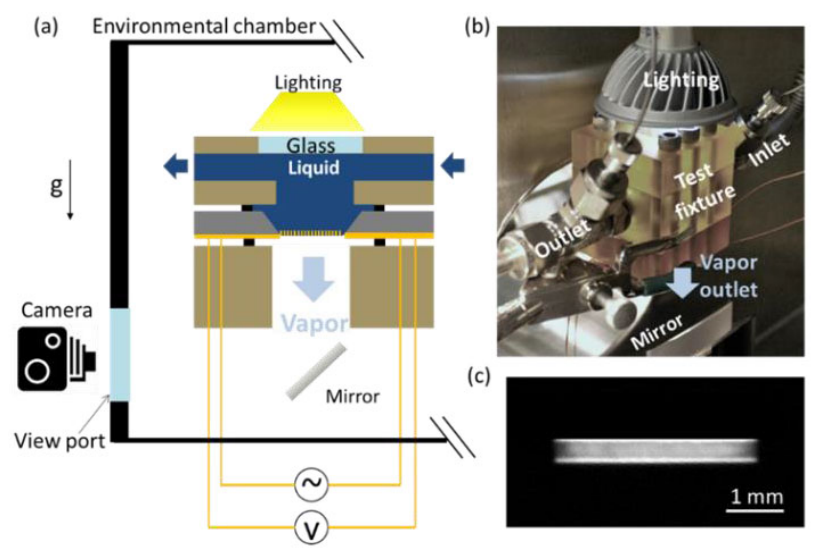

Figure 2: Experimental setup. (a) Schematic showing device placed in a custom test fixture in an environmental chamber which allows for liquid feedthrough, electrical connection, and visualization. (b) Image of experimental setup. (c) Image of the nanoporous membrane device during an experiment.

To characterize the heat loss of the system, we fabricated a control sample with the same structure as the designed device except that the active part was also impermeable. With liquid supplied at 1 $\mathrm{mL} / \mathrm{min}$ (same as in the evaporation experiment), we measured the heating power $Q_{\text {loss }}$ as a function of the temperature rise of the membrane of the control sample $\Delta T$ as the sample was cooled down by convection and conduction. $Q_{\text {loss }}$ is plotted as a function of $\Delta T$ in Figure 3 and the experimental data (red triangles) are fitted to a linear model (black dashed line) $Q_{\text {loss }}=C \Delta T$, which give $C=$ $4.7 \pm 0.1 \mathrm{~mW} / \mathrm{K}$.

We determined the heat loss conductance of the system to be $C$ $=4.7 \pm 0.1 \mathrm{~mW} / \mathrm{K}$. The interfacial heat flux $\dot{q}^{\prime \prime}$ during operation was then recorded as

$$
\dot{q}^{\prime \prime}=\frac{Q-C \Delta T}{A}
$$

where $Q$ is the total Joule heating power, $A$ is the total interface area and $\Delta T=T_{0}-T_{\infty}$, where $T_{\infty}$ is the temperature of the ambient. The heat loss was generally less than $5 \%$ of the total power input in the present study. We characterized evaporation with ambient vapor pressure $P_{\infty}=2.643 \mathrm{kPa}$, and the associated saturation temperature is $T_{\infty}=22.0^{\circ} \mathrm{C}$. Throughout the experiment, the uncertainty in vapor pressure measurement is $\pm 138 \mathrm{~Pa}$ and the one in interface temperature measurement is $\pm 0.52 \mathrm{~K}$.

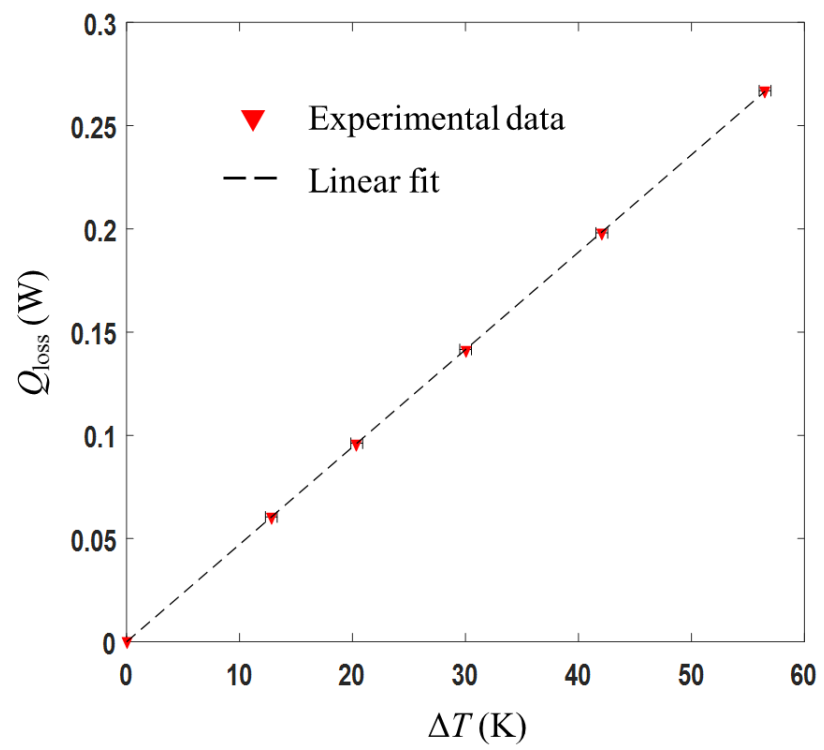

Figure 3 Heat loss characterization using a control sample with impermeable membrane. Qloss is plotted as a function of $\Delta T$ and the experimental data (red triangles) are fitted to a linear model (black dashed line) $Q_{\text {loss }}=C \Delta T$, which give $C=4.7 \pm 0.1 \mathrm{~mW} / \mathrm{K}$.

\section{RESULTS AND DISCUSSION}

The interfacial heat flux $\dot{q}^{\prime \prime}$ is shown in Figure 4 as a function of the temperature rise $\Delta T$. The blue asterisks represent the results from evaporation into an air ambient reported in our previous work [9]. The red diamonds, on the other hand, are the experimental data in the present study of evaporation into a vapor ambient for $T_{\infty}=$ $22.0^{\circ} \mathrm{C}$. Comparing the red diamonds to the green crosses, we note that for similar far field vapor temperatures, the heat transfer coefficient $\left(h=\dot{q}^{\prime \prime} / \Delta T\right)$ associated with evaporation into vapor is much higher than that in the air, because the former is kinetically limited and the latter is diffusion limited. The evaporation data from the present work (red diamonds) show a nearly $10 \times$ improvement from the evaporation into air case (blue asterisks) for the same interface temperature, which is the highest improvement to date.

To properly interpret this trend, we analyzed the nonequilibrium vapor motions in the Knudsen layer using the Boltzmann transport equation (BTE), as the usual thermodynamic quantities are not necessarily well defined under non-equilibrium.

$$
\frac{\partial \xi}{\partial t}=-\mathbf{u} \cdot \nabla_{\mathbf{x}} \xi+\left[\frac{\partial \xi}{\partial t}\right]_{\text {collison }}
$$

where $\xi$ is the mass-based distribution function, $t$ is time, and $\mathbf{u}$ is the molecular velocity. The first term on the right hand side represents the streaming of molecules and the second term is the change of distribution function due to collisions between molecules. The boundary condition at the liquid-vapor interface is characterized by the evaporation coefficient and condensation coefficient ( $\sigma_{\mathrm{e}}$ and $\sigma_{\mathrm{c}}$ ) $[18-20]$. At equilibrium conditions, $\sigma_{\mathrm{e}}=\sigma_{\mathrm{c}}$, analogous to the Kirchhoff's law in thermal radiation [21]. We carried on this equality in our analysis as the Knudsen layer can be considered in near equilibrium for $M_{\mathrm{K}} \ll 1$. The vapor distribution function at the interface $\xi_{0}$ can be written as:

$$
\left.\xi_{0}\right|_{u_{z}>0}=\left[\sigma_{e} \rho_{0}+\left(1-\sigma_{c}\right) \int_{u_{z}<0} \xi_{0} d \mathbf{u}\right] \frac{\exp \left[-\mathbf{u}^{2} / 2 R T_{0}\right]}{\left(2 \pi R T_{0}\right)^{3 / 2}}
$$

where $u_{z}$ is its z-component. The distribution function for the vapor flow coming out of Knudsen layer can be expressed a drifted Maxwell-Boltzmann distribution: 


$$
\left.\xi_{\mathrm{K}}\right|_{u_{z}>0}=\rho_{\mathrm{K}} \frac{\exp \left[-\left(\mathbf{u}-u_{\mathrm{K}} \hat{\mathbf{z}}\right)^{2} / 2 R T_{\mathrm{K}}\right]}{\left(2 \pi R T_{\mathrm{K}}\right)^{3 / 2}}
$$

where $T_{\mathrm{K}}$ is the temperature of the vapor right outside the Knudsen layer. We adopted an evaporation model based on the linearized version of BTE $[18,19]$ using these boundary conditions with $\sigma_{\mathrm{e}}=$ $\sigma_{\mathrm{c}}=1$, which predicts the trend well, but generally overestimates the interfacial heat flux, indicating non-idealities at the interface. It is plotted as orange solid line in Figure 4.

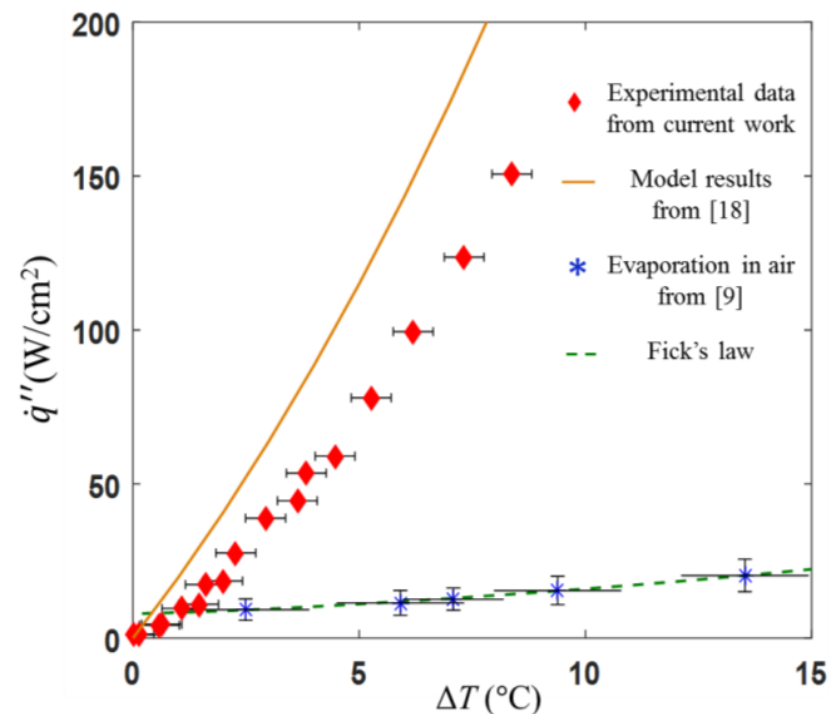

Figure 4: Experimental and modeling results from nanoporous membrane with water as a working fluid. (a) Interfacial heat flux $\dot{q}^{\prime \prime}{ }_{i n}$ as a function of membrane superheat $\Delta T$ for $T_{\infty}=22{ }^{\circ} \mathrm{C}$ : red diamonds represent the evaporation data from the present work; the brown solid line is the model results from [9]; and the blue asterisks and the green dashed line are the experimental data and model prediction for evaporation into air from [18], respectively.

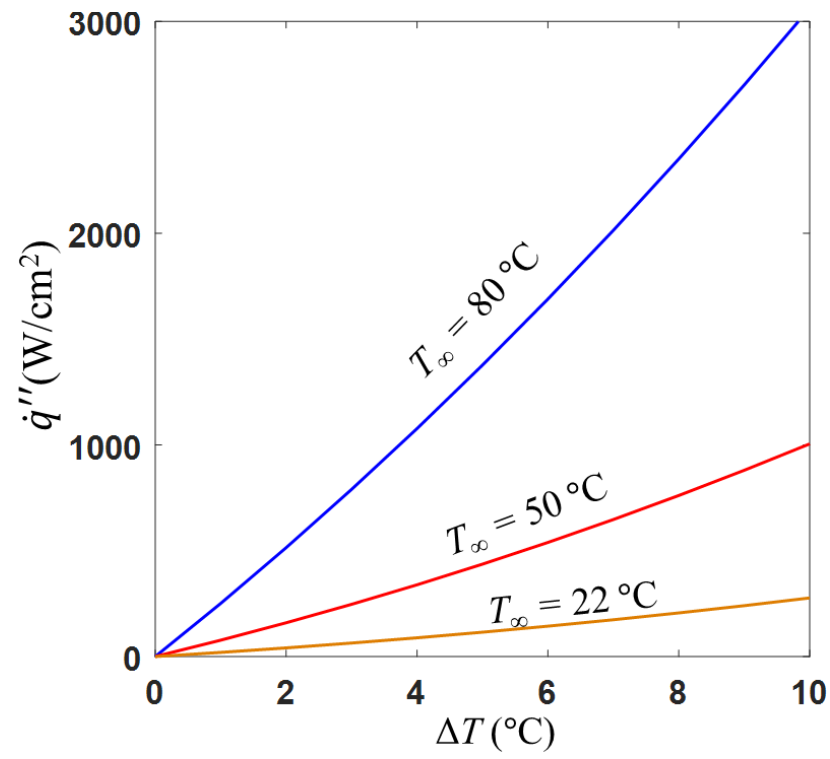

Figure 5: Model prediction of $\dot{q}^{\prime \prime}$ as a function of $\Delta T$ for select $T_{\infty}$ based on the linearized BTE [18]
Moreover, with the trend predicted by this linearized BTE model, by increasing $\mathrm{T}_{\infty}$ while maintaining the superheats, the interfacial heat transfer can be further enhanced shown in Figure 5. The linearized BTE model in fact gives a general figure of merits for evaporative heat transfer: $\rho_{0} u_{s} \Delta h_{l v}$. As the vapor ambient temperature becomes higher, the vapor density increases sharply and so does this figure of merits. This explains why we expect a higher interface heat transfer coefficient for higher vapor ambient temperatures.

\section{CONCLUSIONS}

The ultrathin nanoporous design presented in this study enabled kinetically limited evaporation with non-invasive and accurate interface temperature measurement. With both experiments and modeling, we demonstrated that the kinetic limit of evaporation, enhance the interfacial heat transfer significantly compared to the diffusion-limited evaporation. We generally showed that the interfacial heat transfer coefficient scales with $\rho_{0} u_{\mathrm{s}} \Delta h_{\mathrm{lv}}$ and increases with higher ambient temperatures. This gives the figure of merits for choosing working fluids and operating conditions to maximize evaporative heat transfer. The current work improves the fundamental understanding of the kinetically limited evaporation and shows the significant promise of utilizing an ultrathin nanoporous design to achieve efficient evaporative heat and mass transfer in thermal management, desalination, and steam generation applications, among others. We gratefully acknowledge funding support from the Air Force Office of Scientific Research with Dr. Ali Sayir as program manager.

\section{REFERENCES}

[1] Zhou, L., Tan, Y., Wang, J., Xu, W., Yuan, Y., Cai, W., Zhu, S., and Zhu, J., 2016, "3D self-assembly of aluminium nanoparticles for plasmon-enhanced solar desalination," Nature Photonics, 10(6), p. 393.

[2] Ghasemi, H., Ni, G., Marconnet, A. M., Loomis, J., Yerci, S., Miljkovic, N., and Chen, G., 2014, "Solar steam generation by heat localization," Nature communications, 5, p. ncomms5449.

[3] Lu, Z., Salamon, T. R., Narayanan, S., Bagnall, K. R., Hanks, D. F., Antao, D. S., Barabadi, B., Sircar, J., Simon, M. E., and Wang, E. N., 2016, "Design and modeling of membrane-based evaporative cooling devices for thermal management of high heat fluxes," IEEE Transactions on Components, Packaging and Manufacturing Technology, 6(7), pp. 1056-1065.

[4] Eames, I., Marr, N., and Sabir, H., 1997, "The evaporation coefficient of water: a review," Int. J. Heat Mass Transfer, 40(12), pp. 2963-2973.

[5] Narayanan, S., Fedorov, A. G., and Joshi, Y. K., 2009, "Gasassisted thin-film evaporation from confined spaces for dissipation of high heat fluxes," Nanoscale and Microscale Thermophysical Engineering, 13(1), pp. 30-53.

[6] Weibel, J. A., Garimella, S. V., and North, M. T., 2010, "Characterization of evaporation and boiling from sintered powder wicks fed by capillary action," Int. J. Heat Mass Transfer, 53(19-20), pp. 4204-4215.

[7] Wilke, K. L., Barabadi, B., Lu, Z., Zhang, T., and Wang, E. N., 2017, "Parametric study of thin film evaporation from nanoporous membranes," Appl. Phys. Lett., 111(17), p. 171603.

[8] Xiao, R., Maroo, S. C., and Wang, E. N., 2013, "Negative pressures in nanoporous membranes for thin film evaporation," Appl. Phys. Lett., 102(12), p. 123103.

[9] Lu, Z., Wilke, K. L., Preston, D. J., Kinefuchi, I., ChangDavidson, E., and Wang, E. N., 2017, "An Ultrathin Nanoporous Membrane Evaporator," Nano Lett., 17(10), pp. 6217-6220.

[10] Li, Y., Alibakhshi, M. A., Zhao, Y., and Duan, C., 2017, 
"Exploring Ultimate Water Capillary Evaporation in Nanoscale Conduits," Nano Lett., 17(8), pp. 4813-4819.

[11] Ćoso, D., Srinivasan, V., Lu, M.-C., Chang, J.-Y., and Majumdar, A., 2012, "Enhanced heat transfer in biporous wicks in the thin liquid film evaporation and boiling regimes," J. Heat Transfer, 134(10), p. 101501.

[12] Adera, S., Antao, D., Raj, R., and Wang, E. N., 2016, "Design of micropillar wicks for thin-film evaporation," Int. J. Heat Mass Transfer, 101, pp. 280-294.

[13] Fang, G., and Ward, C., 1999, "Temperature measured close to the interface of an evaporating liquid," PhRvE, 59(1), p. 417.

[14] Lee, J., Laoui, T., and Karnik, R., 2014, "Nanofluidic transport governed by the liquid/vapour interface," Nature nanotechnology, 9(4), p. 317.

[15] Sone, Y., 2000, "Kinetic theoretical studies of the half-space problem of evaporation and condensation," TTSP, 29(3-5), pp. 227-260.

[16] Kundu, P. K., Cohen, I. M., and Dowling, D., 2008, "Fluid Mechanics 4th," Elsevier, Oxford.

[17] Xie, Q., Hong, M., Tan, H., Chen, G., Shi, L., and Chong, T., 2008, "Fabrication of nanostructures with laser interference lithography," J. Alloys Compd., 449(1-2), pp. 261-264.

[18] Frezzotti, A., 2011, "Boundary conditions at the vapor-liquid interface," PhFl, 23(3), p. 030609.

[19] Sone, Y., Takata, S., and Golse, F., 2001, "Notes on the boundary conditions for fluid-dynamic equations on the interface of a gas and its condensed phase," PhFl, 13(1), pp. 324334.

[20] Meland, R., Frezzotti, A., Ytrehus, T., and Hafskjold, B., 2004, "Nonequilibrium molecular-dynamics simulation of net evaporation and net condensation, and evaluation of the gaskinetic boundary condition at the interphase," PhFl, 16(2), pp. 223-243.

[21] Lu, Z., Narayanan, S., and Wang, E. N., 2015, "Modeling of Evaporation from Nanopores with Nonequilibrium and Nonlocal Effects," Langmuir, 31(36), pp. 9817-9824.

\section{CONTACT}

*E.N. Wang, tel: +1-617-324-3311; enwang@mit.edu 